\title{
Multi-Ethnic Comparison of the Characteristics of Amyotrophic Lateral Sclerosis-Related TBK1 Gene Variants
}

\author{
Genki Tohnai ', Ryoichi Nakamura' ${ }^{1}$, Masahiro Nakatochi' ${ }^{2}$, Naoki Atsuta' ${ }^{1}$, Masahisa Katsuno', Gen Sobue ${ }^{1,3 *}$ \\ 'Department of Neurology, Nagoya University Graduate School of Medicine, Nagoya, Japan \\ ${ }^{2}$ Statistical Analysis Section, Center for Advanced Medicine and Clinical Research, Nagoya University Hospital, Nagoya, Japan \\ ${ }^{3}$ Research Division of Dementia and Neurodegenerative Disease, Nagoya University Graduate School of Medicine, Nagoya, Japan
}

Article Info

\section{Article Notes}

Received: December 16, 2018

Accepted: January 29, 2019

\section{*Correspondence:}

Dr. Gen Sobue, Brain and Mind Research Center, and Research Division of Dementia and Neurodegenerative Disease, Nagoya University Graduate School of Medicine, 65 Tsurumai-cho, Showa-ku, Nagoya 466-8550, Japan; Telephone No: $\pm 81-52-744-2794$; Fax No: +81-52-744-2967; Email address: sobueg@med.nagoya-u.ac.jp.

(c) 2019 Sobue $\mathrm{G}$. This article is distributed under the terms of the Creative Commons Attribution 4.0 International License
Abstract

Amyotrophic lateral sclerosis (ALS) is a fatal neurological disease, and the etiology of sporadic ALS is unknown. The TANK-binding kinase 1 (TBK1) gene identified as an ALS gene, contributes towards a predisposition for ALS. In this review, we analyzed variants of TBK1 found in ALS cohort studies belonging to various regions and ethnic populations. TBK1 variants tend to be enriched in patients with ALS compared to patients without ALS. The frequency of TBK1 variants is more in the familial Caucasian than that in the Asian population. However, loss of function (LoF) variant associated with sporadic ALS is almost similar among the Asian group, including Japanese population. LoF variants were frequently reported to be associated with the TBK1 biology. These findings indicate that TBK1- LoF variants are pathogenic for $A L S$, regardless of race or region.

\section{Introduction}

Amyotrophic lateral sclerosis (ALS) is a fatal neurodegenerative disorder characterized by progressive upper and lower motor neuron loss, which causes spasticity and muscle atrophy. Moreover, it leads to general weakness of the skeletal muscles and death within 2-5 years. Approximately $5-10 \%$ of ALS patients have a positive family history, and the rest of them are detected with sporadic ALS ${ }^{1}$.

More than 30 causative genes of familial ALS, including SOD1, FUS, TARDBP, OPTN, and C9ORF72 have been identified. Mutations in these genes can be found in more than $50 \%$ of familial ALS patients, and in a small proportion of sporadic ALS patients ${ }^{2}$. In Japan, a genetic screening study reported that $3 \%$ of sporadic ALS patients have a known familial ALS-related pathogenic gene mutation ${ }^{3}$. To reveal the pathophysiology and develop therapeutics particularly for sporadic ALS, the gene variants and their roles in neurodegeneration in the cohort of sporadic ALS needs to be studied.

TBK1 is a ubiquitously expressed molecule, which plays important roles in multiple cell signaling pathways implicated in autophagy ${ }^{4}$, insulin signaling, and cellular proliferation. TBK1 phosphorylates multiple substrates including optineurin and SQSTM16, which are genes related to ALS. TBK1 was first identified as an ALS related gene through exome sequencing in two independent studies ${ }^{7,8}$. Moreover, the variants of the TBK1 gene in ALS has been reported in the cohort studies of Europe ${ }^{7,8}$, Australia ${ }^{9}$, Taiwan ${ }^{10}$, Belgium ${ }^{11}$, France $^{12}$, China ${ }^{13}$, Korea ${ }^{14}$, Sweden ${ }^{15}$, Germany ${ }^{16}$, Italy ${ }^{17}$, and $\operatorname{Japan}^{18}$. In this review, we summarize the TBK1 variants of each cohort study that has been reported, and their pathogenic significance in ALS. 


\section{TBK1 Structure and Function}

TBK1 is a multifunctional kinase, involved in multiple cellular processes, such as autophagy, cell proliferation, native immune response, and inflammation. TBK1 was initially identified as a kinase mediating the ability of TANK to activate NF-kappa-B (NF-kB) ${ }^{19}$. It is an important member of the I-kappaB (IKB) kinase (IKKs) family and is involved in the regulation of the Interferon regulatory factor 3 (IRF3) and NFKB signaling. The role of TBK1 in selective autophagy has been extensively studied in Salmonella ${ }^{20}$. TBK1 also phosphorylates the ubiquitin-associated (UBA) domain of optineurin on serine-177, enhancing the binding affinity of microtubule-associated protein light chain 3 (LC3) and autophagic clearance of cytosolic Salmonella ${ }^{21,22}$. TBK1 is also involved in the autophagic clearance of Mycobacterium tuberculosis in mouse macrophages and phosphorylates the autophagic receptor SQSTM1 to enhance its binding to polyubiquitinated bacteria ${ }^{23}$. Moreover, TBK1 is particularly important for the maturation of autophagosomes to hydrolytic autophagosomes, which results in degradation of SQSTM1 and its associated cargo ${ }^{5}$.

TBK1 has 729 amino acids, a serine/threonine kinase domain, a ubiquitin-like domain (ULD), and two c-terminal coiled-coil domains (CCD1 and CCD2). The serine/threonine kinase domain phosphorylates various substrates such as the interferon regulatory factor $3^{24}$. The ULD region of the TBK1 regulates the kinase activity and interaction with other molecules in the TBK1 pathway ${ }^{25}$. The CCD region of TBK1 binds to optineurin and phosphorylates SQSTM ${ }^{6,8}$.

\section{TBK1 variants in the ALS and Control Cohorts}

\section{Racial frequency of $T B K 1$ variants}

TBK1 was first reported as a causative ALS gene by two independent studies ${ }^{7,8}$. Cirulli et al. analyzed TBK1 variants using a number of inheritance models by performing exome sequencing on 2869 ALS patients and genetically on Caucasian ethnic healthy controls ${ }^{7}$. This study confirmed several previously identified ALS genes and reported TBK1 as a new ALS-related gene. Freischmidt et al. identified TBK1 as an ALS gene using the exome sequencing method and the target variant screen in Swedish population ${ }^{8}$. Moreover, the study conducted on an Australian cohort of familial ALS patients identified a novel $T B K 1 \mathrm{LoF}$ variant in a family of Chinese origin ${ }^{9}$. Subsequently, the TBK1 LoF variant was found to be a rare cause of ALS in the Taiwanese ${ }^{10}$ and Chinese cohort $^{13}$, as well as in Sardinian ALS patients ${ }^{15}$. TBK1 LoF variants have been reported in more than 10 cohorts so far (Table 1), including approximately $1.6 \%$ patients with familial ALS and $0.4 \%$ patients with sporadic ALS. Patients with familial ALS have more TBK1 LoF variants than sporadic ALS patients, probably because they include family members with LoF variants in the cohorts examined as well. In our previous study, we analyzed the TBK1 gene by exome sequencing in a large Japanese cohort of 713 sporadic ALS patients and 800 controls $^{18}$. All cases were registrant of Japanese Consortium for Amyotrophic Lateral Sclerosis Research (JaCALS) ${ }^{3,26}$. We identified LoF variants of TBK1 in $0.42 \%$ (3/713) sporadic ALS patients in our cohort.

A meta-analysis by Cui et al., revealed that the frequency of TBK1 variants is higher in Caucasian patients than in Asians ${ }^{27}$. Therefore, we summarized the data of $T B K 1$ variants in each cohort including recent studies (Table 1). The frequency of missense variants in the TBK1 gene was higher in Caucasian sporadic ALS patients than in Asians ${ }^{7}$. However, there was no racial difference in the frequency of TBK1 LoF variants in cohort studies with sporadic ALS (Caucasian sporadic ALS patients: $0.44 \%$; Asian patients: $0.39 \%$ ). On the contrary, the TBK1 LoF variants in the familial ALS patients were reported only in Caucasian patients (Caucasian familial ALS patients: 1.69\%; Asian patients: 0\%).

Table 1. Characteristics of TANK-binding kinase 1 (TBK1) variants in the cohort

\begin{tabular}{|c|c|c|c|c|c|c|c|c|c|}
\hline \multirow[b]{2}{*}{ Population } & \multirow[b]{2}{*}{\begin{tabular}{|c|} 
LoF \\
variants
\end{tabular}} & \multirow[b]{2}{*}{$\begin{array}{l}\text { Missense } \\
\text { variants }\end{array}$} & \multicolumn{3}{|c|}{ Frequency of LoF variants } & \multirow[b]{2}{*}{ Patients } & \multirow[b]{2}{*}{ Cohort } & \multirow[b]{2}{*}{ Year } & \multirow[b]{2}{*}{ Reference } \\
\hline & & & fALS patients & sALS patients & Total ALS patients & & & & \\
\hline Caucasian & 8 & 33 & - & - & $0.28 \%(8 / 2874)$ & 2874 (fALS105/sALS1563) & European & 2015 & 7 \\
\hline Caucasian & 12 & 100 & $3.5 \%(9 / 252)$ & $0.28 \%(3 / 1010)$ & $0.95 \%(11 / 1262)$ & 1262(fALS252/sALS1010) & European & 2015 & 8 \\
\hline Caucasian & 1 & 0 & $0.79 \%(1 / 127)$ & - & $0.79 \%(1 / 127)$ & 127(fALS) & Australian & 2015 & 9 \\
\hline Caucasian & 5 & 5 & - & - & $1.2 \%(5 / 416)$ & 416 & French & 2015 & 12 \\
\hline Caucasian & 11 & 5 & - & - & $1.75 \%(11 / 629)$ & 629(fALS154/sALS475) & Belgian & 2015 & 11 \\
\hline Asian & 0 & 2 & $0(0 / 23)$ & $0(0 / 271)$ & $0(0 / 294)$ & 294(fALS23/sALS271) & Chinese & 2016 & 13 \\
\hline Asian & 1 & 2 & $0(0 / 32)$ & $0.57 \%(1 / 175)$ & $0.48 \%(1 / 207)$ & 207(sALS175) & Taiwanese & 2016 & 10 \\
\hline Asian & 1 & 3 & - & $0.78 \%(1 / 129)$ & $0.78 \%(1 / 129)$ & 129(sALS) & Korean & 2016 & 14 \\
\hline Caucasian & 1 & 2 & - & - & $0.54 \%(1 / 186)$ & 186 & Sardinian & 2016 & 15 \\
\hline Caucasian & 2 & 5 & $0(0 / 30)$ & $1.6 \%(2 / 124)$ & $1.3 \%(2 / 154)$ & 154(fALS30/sALS124) & Italian & 2017 & 17 \\
\hline Asian & 3 & 6 & - & $0.42 \%(3 / 713)$ & $0.42 \%(3 / 713)$ & 713(sALS) & Japanese & 2018 & 18 \\
\hline Caucasian & 2 & 2 & $0.66 \%(2 / 301)$ & - & $0.66 \%(2 / 301)$ & 301(fALS) & German & 2018 & 16 \\
\hline
\end{tabular}

Key: ALS: amyotrophic lateral sclerosis, sALS: sporadic ALS, fALS: familial ALS, LoF: loss-of-function 
Missense variants

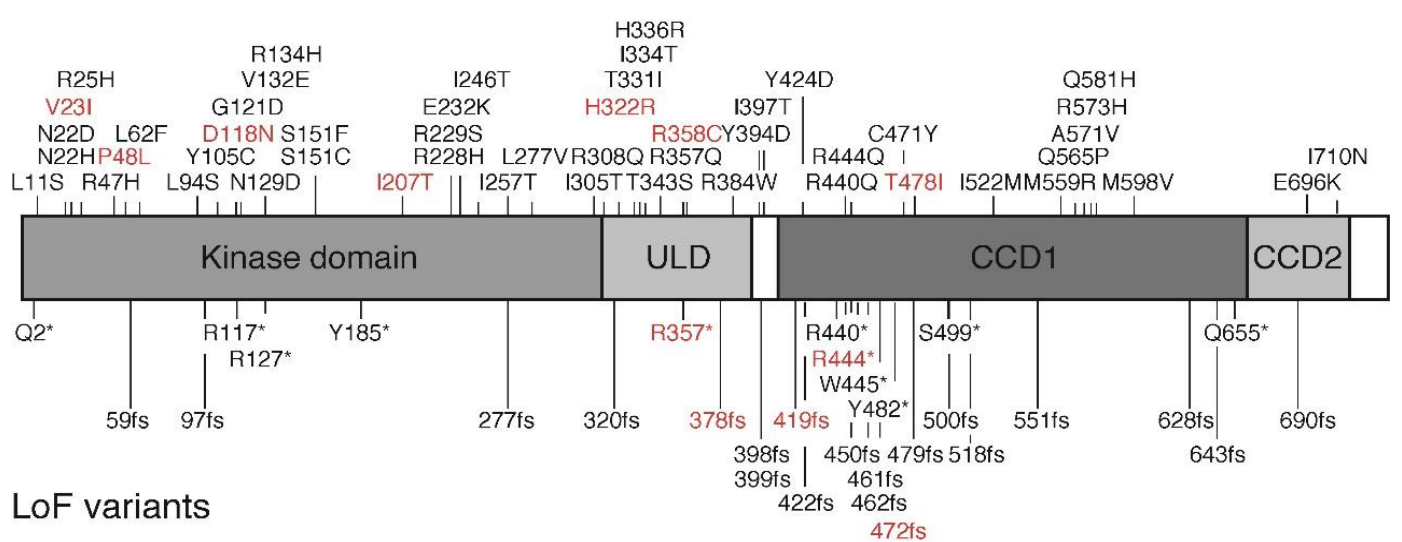

\section{Figure. Schematic diagram showing the location of TBK1 variants}

Schematic representation of the location of missense variants (above) and LoF variants (below). Red letters indicate variants found in East Asia and black letters indicate those found in Europe. Abbreviations: CCD, C-terminal coiled-coil domain; ULD, ubiquitin-like domain.

*: a terminal codon, fs: frameshift (leading to a premature terminal codon)

Table 2. Clinical characteristics of Asian ALS patients with LoF variant of the TBK1 gene

\begin{tabular}{|l|c|c|c|c|c|c|}
\hline Variant & Sex & $\begin{array}{c}\text { Age at } \\
\text { onset }\end{array}$ & Site and symptoms of onset & $\begin{array}{c}\text { Cognitive } \\
\text { impairment }\end{array}$ & TPPV free survival duration (Month) & Population \\
\hline p.Arg357Term & Male & 49.0 & Dysarthria & - & 8 & Japanese \\
\hline $378 f s$ & Female & 55.9 & Dysarthria & - & $>20$ & Japanese \\
\hline $419 \mathrm{fs}$ & Male & 62.2 & Lower Limb & - & $>10$ & Japanese \\
\hline p.Arg444Term & Female & 55.0 & Upper Limb + Behaviour disorder & + & Died at 59 years old & Taiwanese \\
\hline $472 \mathrm{fs}$ & Male & 53.0 & Dysarthria+Dysphagia & - & Gastrostomy at 46 months after onset & Korean \\
\hline
\end{tabular}

TPPV; Tracheostomy positive pressure ventilation

\section{Distribution of variants in the TBK1 molecule, and their pathogenicity}

TBK1 mutation has been found in the TBK1 gene from various patients. Thus, we evaluated the location of the TBK1 variants including the LoF variants in TBK1 gene among Caucasian and Asian patients (Figure). We could not find remarkable racial differences in TBK1 missense variants between Asian and Caucasian ALS patients. The LoF variants of TBK1 were distributed throughout the gene in Caucasian ALS patients. On the contrary, LoF variants of TBK1 were present around the ULD domain in Asian ALS patients. The clinical manifestation of Asian ALS patients with TBK1 LoF variant varies in age and site of onset, neuropsychological abnormalities, and survival (Table 2). Freischmidt et al. identified eight LoF variants, seven of which were linked to the loss of expression of TBK1 and were therefore reported as a causative via haploinsufficiency ${ }^{8}$. TBK1 variants were enriched in the cohorts compared to controls. In our study, the frequency of TBK1 variants was higher in ALS patients than in healthy controls $(\mathrm{OR}=10.2 ; \mathrm{p}=0.008,95 \%$ confidence interval $=1.67-62.47)$. Moreover, patients with variants of $T B K 1$ had an earlier onset age than those without TBK1 variants in our study ${ }^{18}$. A majority of currently identified ALS-related TBK1 variants are unknown pathogenic missense variants ${ }^{28}$. However, many of the missense variants have not been elucidated for their pathogenicity. The pathogenicity of the missense variants can be predicted by in silico analysis using several algorithms (eg. SIFT (http://sift.jcvi.org/), PolyPhen-2 software (http:// genetics.bwh.harvard.edu/pph2/), and CADD $^{29}$ etc.). Freischmidt et al., suggested that TBK1 missense variants can cause TBK1 dysfunction in vitro ${ }^{8}$, demonstrating that variants in the CDD domain hindered the binding of TBK1 to optineurin. Xu et al, reported that decreased myeloid TAK1 (transforming growth factor- $\beta$-activated kinase 1) expression in TBK1 knockdown mice promotes ALS / FTD features including neuroinflammation, TDP43 aggregation, axonal degeneration, neuronal loss, and behavioral disturbance ${ }^{30}$. This result indicates that reduction of TAK1 expression in the brain due to aging and mutation of TBK1 are sufficient to produce important features of ALS / FTD. However, only a few missense variants were verified for their pathogenicity. Further evidence is needed to classify the missense variants as pathogenic or non-pathogenic. 


\section{Clinical manifestation of TBK1 variants}

The clinical manifestation of ALS patients with TBK1 LoF variant varies in age and site of onset, neuropsychological abnormalities, and survival. The age of onset ranges from 30 to 80 years, and survival ranges from a few months to more than 10 years. Some patients show extrapyramidal symptoms such as positional tremor, bradykinesia ${ }^{31}$, primary lateral sclerosis ${ }^{32}$, progressive supranuclear palsy (PSP)-like syndrome, and cerebellar ataxia ${ }^{33}$. Brain MRI findings of ALS patients with the mutation of the TBK1 gene are variable, although the temporal lobe atrophy is the most frequently involved $^{31}$. The fluorodeoxyglucose positron emission tomography (FDG-PET) in ALS-FTD patients indicated that glucose metabolism decreased in the frontal and temporal lobe. Immunohistochemistry of the brain tissue and spinal cord from patients with a TBK1 LoF variant showed TDP-43positve and p62-positive neuronal cytoplasmic inclusions, as well as ALS patients without TBK1 LoF variants ${ }^{11,31,34}$. Here, one patient carrying the TBK1 missense variant was diagnosed with ALS-FTD, and the patient showed alterations of behavior preceding the appearance of motor symptoms and atrophy of the temporal lobes ${ }^{18}$.

\section{Conclusion}

TBK1 variants tend to be enriched in the cohort compared to the control. There is no racial difference in the frequency of TBK1 LoF variants analyzed in the cohort studies for sporadic ALS patients. Variants of TBK1 were scattered throughout the genes of ALS patients, and there were no racial differences in the missense variants. However, in Asian patients, accumulation of LoF variants around the ULD region was observed. The accumulation may be due to the small number of cases as compared with those of Caucasian cohorts. In the future, as research progresses, a similar result might be observed in Caucasian patients. The LoF variants of TBK1 are likely pathogenic for sporadic ALS, regardless of race or region, however, the pathogenicity of missense variants of $T B K 1$ is somewhat difficult. Further studies are necessary to investigate pathogenicity of individual missense variants through molecular biological techniques.

\section{Acknowledgments}

This study was supported by the Practical Research Project for Rare/Intractable Diseases (16ek0109071h0003) from the Japan Agency for Medical Research and Development (AMED) and Health and Labor Sciences Research grants, Grants-in-Aid for Young Scientists (B) $(17 \mathrm{~K} 16116,17 \mathrm{~K} 16118)$ from the Japan Society for the Promotion of Science (JSPS).

\section{References}

1. Rowland LP, Shneider NA. Amyotrophic lateral sclerosis. N Engl J Med 2001; 344: 1688-1700.
2. Renton AE, Chio A, Traynor BJ. State of play in amyotrophic lateral sclerosis genetics. Nat Neurosci. 2014; 17: 17-23.

3. Nakamura R, Sone J, Atsuta N, et al. Next-generation sequencing of 28 ALS-related genes in a Japanese ALS cohort. Neurobiol Aging. 2016; 39: 219 e211-218.

4. Stolz A, Ernst A, Dikic I. Cargo recognition and trafficking in selective autophagy. Nature cell biology. 2014; 16: 495-501.

5. Fitzgerald KA, McWhirter SM, Faia KL, et al. IKKepsilon and TBK1 are essential components of the IRF3 signaling pathway. Nature immunology. 2003; 4: 491-496.

6. Ahmad L, Zhang SY, Casanova JL, et al. Human TBK1: A Gatekeeper of Neuroinflammation. Trends in molecular medicine. 2016; 22: 511527.

7. Cirulli ET, Lasseigne BN, Petrovski S, et al. Exome sequencing in amyotrophic lateral sclerosis identifies risk genes and pathways. Science. 2015; 347: 1436-1441.

8. Freischmidt A, Wieland T, Richter B, et al. Haploinsufficiency of TBK1 causes familial ALS and fronto-temporal dementia. Nat Neurosci. 2015; 18: 631-636.

9. Williams KL, McCann EP, Fifita JA, et al. Novel TBK1 truncating mutation in a familial amyotrophic lateral sclerosis patient of Chinese origin. Neurobiol Aging. 2015; 36: 3334 e3331-3334 e3335.

10. Tsai PC, Liu YC, Lin KP, et al. Mutational analysis of TBK1 in Taiwanese patients with amyotrophic lateral sclerosis. Neurobiol Aging. 2016; 40: 191 e111-191 e116.

11. Gijselinck I, Van Mossevelde S, van der Zee J, et al. Loss of TBK1 is a frequent cause of frontotemporal dementia in a Belgian cohort. Neurology. 2015; 85: 2116-2125.

12. Le Ber I, De Septenville A, Millecamps S, et al. TBK1 mutation frequencies in French frontotemporal dementia and amyotrophic lateral sclerosis cohorts. Neurobiol Aging. 2015 Nov; 36(11): 3116. e5-3116.e8.

13. Shu S, Li XL, Liu Q et al. Screening of the TBK1 gene in familial and sporadic amyotrophic lateral sclerosis patients of Chinese origin. Amyotrophic lateral sclerosis \& frontotemporal degeneration. 2016; 17: 605-607.

14. Kim YE, Oh KW, Noh MY, et al. Genetic and functional analysis of TBK1 variants in Korean patients with sporadic amyotrophic lateral sclerosis. Neurobiol Aging. 2017 Feb; 50: 170.e1-170.e6.

15. Borghero G, Pugliatti M, Marrosu F, et al. TBK1 is associated with ALS and ALS-FTD in Sardinian patients. Neurobiol Aging. 2016; 43: 180 e181-185.

16. Müller K, Brenner D, Weydt $P$, et al. Comprehensive analysis of the mutation spectrum in 301 German ALS families. J Neurol Neurosurg Psychiatry. 2018 Aug; 89(8): 817-827

17. Pozzi L, Valenza F, Mosca L, et al. TBK1 mutations in Italian patients with amyotrophic lateral sclerosis: genetic and functional characterisation. J Neurol Neurosurg Psychiatry. 2017 Oct; 88(10): 869-875.

18. Tohnai G, Nakamura R, Sone J, et al. Frequency and characteristics of the TBK1 gene variants in Japanese patients with sporadic amyotrophic lateral sclerosis. Neurobiol Aging. 2018; 64: 158 e115158 e119.

19. Pomerantz JL, Baltimore D. NF-kappaB activation by a signaling complex containing TRAF2, TANK and TBK1, a novel IKK-related kinase. ЕMBO J. 1999; 18: 6694-6704.

20. Thurston TL, Ryzhakov G, Bloor S, et al. The TBK1 adaptor and autophagy receptor NDP52 restricts the proliferation of ubiquitincoated bacteria. Nature immunology. 2009; 10: 1215-1221. 
21. Nguyen DKH, Thombre R, Wang J. Autophagy as a common pathway in amyotrophic lateral sclerosis. Neurosci Lett. 2018.

22. Wild P, Farhan H, McEwan DG, et al. Phosphorylation of the autophagy receptor optineurin restricts Salmonella growth. Science. 2011; 333: 228-233.

23. Pilli M, Arko-Mensah J, Ponpuak M, et al. TBK-1 promotes autophagymediated antimicrobial defense by controlling autophagosome maturation. Immunity. 2012; 37: 223-234.

24. Sharma S, tenOever BR, Grandvaux N, et al. Triggering the interferon antiviral response through an IKK-related pathway. Science. 2003; 300: 1148-1151.

25. Li J, Li J, Miyahira A, et al. Crystal structure of the ubiquitin-like domain of human TBK1. Protein \& cell. 2012; 3: 383-391.

26. Watanabe $\mathrm{H}$, Atsuta N, Hirakawa A, et al. A rapid functional decline type of amyotrophic lateral sclerosis is linked to low expression of TTN. J Neurol Neurosurg Psychiatry. 2016 Aug; 87(8): 851-857

27. Cui R, Tuo M1, Li P, et al. Association between TBK1 mutations and risk of amyotrophic lateral sclerosis/frontotemporal dementia spectrum: a meta-analysis. Neurol Sci. 2018 May; 39(5): 811-820.

28. Freischmidt A, Muller K, Ludolph AC, et al. Association of Mutations in
TBK1 With Sporadic and Familial Amyotrophic Lateral Sclerosis and Frontotemporal Dementia. JAMA neurology. 2017; 74: 110-113.

29. Kircher M, Witten DM, Jain P, et al. A general framework for estimating the relative pathogenicity of human genetic variants. Nat Genet. 2014; 46: 310-315.

30. Xu D, Jin T, Zhu H, et al. TBK1 Suppresses RIPK1-Driven Apoptosis and Inflammation during Development and in Aging. Cell. 2018 Sep 6; 174(6): 1477-1491.

31. Van Mossevelde S, van der Zee J, Gijselinck I, et al. Clinical features of TBK1 carriers compared with C9orf72, GRN and non-mutation carriers in a Belgian cohort. Brain. 2016; 139: 452-467.

32. Gomez-Tortosa E, Van der Zee J, Ruggiero M, et al. Familial primary lateral sclerosis or dementia associated with Arg573Gly TBK1 mutation. J Neurol Neurosurg Psychiatry. 2017; 88: 996-997.

33. Wilke C, Baets J, De Bleecker JL, et al. Beyond ALS and FTD: the phenotypic spectrum of TBK1 mutations includes PSP-like and cerebellar phenotypes. Neurobiol Aging. 2018; 62: 244 e249-244 e213.

34. van der Zee J, Gijselinck I, Van Mossevelde S, et al. TBK1 Mutation Spectrum in an Extended European Patient Cohort with Frontotemporal Dementia and Amyotrophic Lateral Sclerosis. Hum Mutat. 2017; 38: 297-309. 